\title{
Followers' Strategy in Stackelberg Equilibrium Problems on Curved Strategy Sets
}

\author{
Alexandru Kristály \\ Óbuda University, Institute of Applied Mathematics, Budapest, Hungary \\ Babeş-Bolyai University, 400591 Cluj-Napoca, Romania \\ Email address: alexandru.kristaly@econ.ubbcluj.ro
}

\section{Szilárd Nagy}

Babeş-Bolyai University, 400591 Cluj-Napoca, Romania

Email address: szilard.nagy@econ.ubbcluj.ro

Abstract: We study the existence of Stackelberg equilibrium points on strategy sets which are geodesic convex in certain Riemannian manifolds by using metric projection arguments. The present results extend those obtained in Nagy [J. Global Optimization (2013)] in the Euclidean context.

Keywords: Stackelberg model; curved spaces; variational analysis

\section{Introduction}

Recently, the second author obtained certain existence and location results for the Stackelberg equilibria in the Euclidean framework, see [9]. More precisely, the existence of solutions for the leader-follower game has been obtained via the study of certain variational inequalities defined on the strategy sets by using the variational backward induction method.

The purpose of the present study is to extend the analytical results from [9] to games defined on strategy sets which are embedded in a geodesic convex manner into certain Riemannian manifolds. Similar studies can be found in the literature, where certain variational arguments are applied to study equilibrium problems on Riemannian manifolds, see [4], [7], [11], [10] and references therein.

For simplicity, in the present paper we shall consider only two players although our arguments can be extended to several players as well. Let $K_{1} \subset M_{1}$ and $K_{2} \subset M_{2}$ be two sets in the Riemannian manifolds $\left(M_{1}, g_{1}\right)$ and $\left(M_{2}, g_{2}\right)$, respectively, and let $h_{1}, h_{2}: M_{1} \times M_{2} \rightarrow \mathbf{R}$ be the payoff functions for the two players. As we already 
know from the backward induction method, the first step (for the follower) is to find the response set

$$
\mathscr{R}_{S E}\left(x_{1}\right)=\left\{x_{2} \in K_{2}: h_{2}\left(x_{1}, y\right)-h_{2}\left(x_{1}, x_{2}\right) \geq 0, \forall y \in K_{2}\right\}
$$

for every fixed $x_{1} \in K_{1}$. If $\mathscr{R}_{S E}\left(x_{1}\right) \neq \emptyset$ for every $x_{1} \in K_{1}$, the next step (for the leader) is to minimize the map $x \mapsto h_{1}(x, r(x))$ on $K_{1}$ where $r$ is a fixed selection function of the set-valued map $x \mapsto \mathscr{R}_{S E}(x)$; more precisely, the objective of the first player is to determine the set

$$
\mathscr{S}_{S E}=\left\{x_{1} \in K_{1}: h_{1}(x, r(x))-h_{1}\left(x_{1}, r\left(x_{1}\right)\right) \geq 0, \forall x \in K_{1}\right\} .
$$

Since the location of the sets $\mathscr{R}_{S E}\left(x_{1}\right)$ and $\mathscr{S}_{S E}$ is not an easy task, we shall introduce further sets related to them by variational inequalities defined on the Riemannian manifolds. Let us assume that $h_{2}: M_{1} \times M_{2} \rightarrow \mathbf{R}$ is a function of class $C^{1}$; for every $x_{1} \in K_{1}$, we introduce the set

$$
\mathscr{R}_{S V}\left(x_{1}\right)=\left\{x_{2} \in K_{2}: g_{2}\left(\frac{\partial h_{2}}{\partial x_{2}}\left(x_{1}, x_{2}\right), \exp _{x_{2}}^{-1}(y)\right) \geq 0, \forall y \in K_{2}\right\} .
$$

Here and in the sequel, exp denotes the usual exponential function in Riemannian geometry. According to [4] and [5], it is more easier to determine the set $\mathscr{R}_{S V}\left(x_{1}\right)$ than $\mathscr{R}_{S E}\left(x_{1}\right)$. Moreover, usually we have that $\mathscr{R}_{S E}\left(x_{1}\right) \subset \mathscr{R}_{S V}\left(x_{1}\right)$, thus we shall choose the appropriate Stackelberg equilibrium candidates from the elements of the latter set. Finally, by imposing further curvature assumptions on the Riemannian manifolds we are working on, we are able to characterize the elements of the set $\mathscr{R}_{S V}\left(x_{1}\right)$ by the fixed points of a suitable set-valued map which involves the metric projection map into the set $K_{2}$. In fact, we shall assume that the strategy sets are embedded into non-positively curved Riemannian manifolds where two basic properties of the metric projection will be deeply exploited; namely, the non-expansiveness and the so-called Moskovitz-Dines property (see [8]); for further details, see Section 2. Having this fixed-point characterization, we will be able to apply various fixed point theorems on (acyclic) metric spaces in order to find elements of the set $\mathscr{R}_{S V}\left(x_{1}\right)$. We emphasize that projection-like methods for Nash equilibria have been developed in the Euclidean context in [1], [15], [16].

We assume finally that $h_{1}: M_{1} \times M_{2} \rightarrow \mathbf{R}$ is a function of class $C^{1}$ and for every $x_{1} \in K_{1}$ we have that $\mathscr{R}_{S V}\left(x_{1}\right) \neq \emptyset$. If we are able to choose a $C^{1}$-class selection $r: K_{1} \rightarrow K_{1}$ of the set-valued map $\mathscr{R}_{S V}$, we also introduce the set

$$
\mathscr{S}_{S V}=\left\{x_{1} \in K_{1}: g_{1}\left(\frac{\partial h_{1}}{\partial x_{1}}\left(x_{1}, r\left(x_{1}\right)\right), \exp _{x_{1}}^{-1}(y)\right) \geq 0, \forall y \in K_{1}\right\} .
$$

In particular, $\mathscr{S}_{S V}$ contains the optimal strategies of the leader, i.e., the minimizers for the map $x \mapsto h_{1}(x, r(x))$ on $K_{1}$.

Section 2 contains some basic notions and results from Riemannian geometry which are needed for our investigations: geodesics, curvature, metric projections, MoskovitzDines property, etc. Finally, in Section 3 we present the main results of the paper concerning the strategy of the follower. 


\section{Preliminaries}

\subsection{Elements from Riemannian manifolds}

Let $(M, g)$ be a connected $m$-dimensional Riemannian manifold, $m \geq 2$, and let $T M=\cup_{p \in M}\left(p, T_{p} M\right)$ and $T^{*} M=\cup_{p \in M}\left(p, T_{p}^{*} M\right)$ be the tangent and cotangent bundles to $M$. If $\xi \in T_{p}^{*} M$ then there exists a unique $W_{\xi} \in T_{p} M$ such that

$\langle\xi, V\rangle_{g, p}=g_{p}\left(W_{\xi}, V\right)$ for all $V \in T_{p} M$.

Due to (1), the elements $\xi$ and $W_{\xi}$ are identified. The norms on $T_{p} M$ and $T_{p}^{*} M$ are defined by

$$
\|\xi\|_{g}=\left\|W_{\xi}\right\|_{g}=\sqrt{g\left(W_{\xi}, W_{\xi}\right)} .
$$

It is clear that for every $V \in T_{p} M$ and $\xi \in T_{p}^{*} M$,

$\left|\langle\xi, V\rangle_{g}\right| \leq\|\xi\|_{g}\|V\|_{g}$

Let $h: M \rightarrow \mathbf{R}$ be a $C^{1}$ function at $p \in M$; the differential of $h$ at $p$, denoted by $d h(p)$, belongs to $T_{p}^{*} M$ and is defined by

$$
\langle d h(p), V\rangle_{g}=g(\operatorname{grad} h(p), V) \text { for all } V \in T_{p} M .
$$

Let $\gamma:[0, r] \rightarrow M$ be a $C^{1}$ path, $r>0$. The length of $\gamma$ is defined by $L_{g}(\gamma)=$ $\int_{0}^{r}\|\dot{\gamma}(t)\|_{g} d t$. For any two points $p, q \in M$, let

$$
d_{g}(p, q)=\inf \left\{L_{g}(\gamma): \gamma \text { is a } C^{1} \text { path joining } p \text { and } q \text { in } M\right\} .
$$

The function $d_{g}: M \times M \rightarrow \mathbf{R}$ clearly verifies the properties of the metric function. For every $p \in M$ and $r>0$, the open ball of center $p \in M$ and radius $r>0$ is defined by

$$
B_{g}(p, r)=\left\{q \in M: d_{g}(p, q)<r\right\}
$$

A $C^{\infty}$ parameterized path $\gamma$ is a geodesic in $(M, g)$ if its tangent $\dot{\gamma}$ is parallel along itself, i.e., $\nabla_{\dot{\gamma}} \dot{\gamma}=0$. Here, $\nabla$ is the Levi-Civita connection. The geodesic segment $\gamma:[a, b] \rightarrow M$ is called minimizing if $L_{g}(\gamma)=d_{g}(\gamma(a), \gamma(b))$. From the theory of ODE we have that for every $V \in T_{p} M, p \in M$, there exists an open interval $I_{V} \ni 0$ and a unique geodesic $\gamma_{V}: I_{V} \rightarrow M$ with $\gamma_{V}(0)=p$ and $\dot{\gamma}_{V}(0)=V$. On account of [2, p. 64], we introduce the exponential map $\exp _{p}: T_{p} M \rightarrow M$ as $\exp _{p}(V)=\gamma_{V}(1)$. Moreover,

$$
d \exp _{p}(0)=\operatorname{id}_{T_{p} M} .
$$

In particular, for every two points $q_{1}, q_{2} \in M$ which are close enough to each other, we have

$\left\|\exp _{q_{1}}^{-1}\left(q_{2}\right)\right\|_{g}=d_{g}\left(q_{1}, q_{2}\right)$.

Let $K \subset M$ be a non-empty set. Let

$$
P_{K}(q)=\left\{p \in K: d_{g}(q, p)=\inf _{z \in K} d_{g}(q, z)\right\}
$$


be the set of metric projections of the point $q \in M$ to the set $K$. According to the theorem of Hopf-Rinow, if $(M, g)$ is complete, then for any closed set $K \subset M$ we have that $\operatorname{card}\left(P_{K}(q)\right) \geq 1$ for every $q \in M$. The map $P_{K}$ is non-expansive if

$$
d_{g}\left(p_{1}, p_{2}\right) \leq d_{g}\left(q_{1}, q_{2}\right) \text { for all } q_{1}, q_{2} \in M \text { and } p_{1} \in P_{K}\left(q_{1}\right), p_{2} \in P_{K}\left(q_{2}\right) .
$$

In particular, when $P_{K}$ is non-expansive, then $K$ is a Chebishev set, i.e., $\operatorname{card}\left(P_{K}(q)\right)=$ 1 for every $q \in M$.

The set $K \subset M$ is geodesic convex if every two points $q_{1}, q_{2} \in K$ can be joined by a unique minimizing geodesic whose image belongs to $K$. Clearly, relation (3) holds for every $q_{1}, q_{2} \in K$ in a geodesic convex set $K$ since $\exp _{q_{i}}^{-1}$ is well-defined on $K$, $i \in\{1,2\}$. The function $f: K \rightarrow \mathbf{R}$ is convex, if $f \circ \gamma:[0,1] \rightarrow \mathbf{R}$ is convex in the usual sense for every geodesic $\gamma:[0,1] \rightarrow K$ once $K \subset M$ is a geodesic convex set.

A non-empty closed set $K \subset M$ verifies the Moskovitz-Dines property if for fixed $q \in M$ and $p \in K$ the following two statements are equivalent:

$\left(M D_{1}\right) p \in P_{K}(q)$

$\left(M D_{2}\right)$ If $\gamma:[0,1] \rightarrow M$ is the unique minimal geodesic from $\gamma(0)=p \in K$ to $\gamma(1)=$ $q$, then for every geodesic $\sigma:[0, \delta] \rightarrow K(\delta \geq 0)$ emanating from the point $p$, we have $g(\dot{\gamma}(0), \dot{\sigma}(0)) \leq 0$.

A Riemannian manifold $(M, g)$ is a Hadamard manifold if it is complete, simply connected and its sectional curvature is non-positive. We recall that on a Hadamard manifold $(M, g)$, if $h(p)=d_{g}^{2}\left(p, p_{0}\right), p_{0} \in M$ is fixed, then

$\operatorname{grad} h(p)=-2 \exp _{p}^{-1}\left(p_{0}\right)$

It is well-known that on a Hadamard manifold $(M, g)$ every geodesic convex set is a Chebyshev set. Moreover, we have

Proposition 1. ([3], [13]) Let $(M, g)$ be a finite-dimensional Hadamard manifold, $K \subset M$ be a closed set. The following statements hold true:

(i) If $K \subset M$ is geodesic convex, it verifies the Moskovitz-Dines property;

(ii) $P_{K}$ is non-expansive if and only if $K \subset M$ is geodesic convex.

\subsection{Basic properties of the response sets}

In the sequel we shall establish some basic properties of the response sets by using some elements from the theory of variational inequalities on Riemannian manifolds.

Lemma 1. Let $\left(M_{i}, g_{i}\right)$ be Riemannian manifolds, $h_{i}: M_{1} \times M_{2} \rightarrow \mathbf{R}$ be functions of class $C^{1}$, and $K_{i} \subset M_{i}$ closed, geodesic convex sets, $i=1,2$. Then the following assertions hold:

(i) $\mathscr{R}_{S E}\left(x_{1}\right) \subseteq \mathscr{R}_{S V}\left(x_{1}\right)$ for every $x_{1} \in K_{1}$;

(ii) $\mathscr{R}_{S E}\left(x_{1}\right)=\mathscr{R}_{S V}\left(x_{1}\right)$ when $h_{2}\left(x_{1}, \cdot\right)$ is convex on $K_{2}$ for some $x_{1} \in K_{1}$; 
(iii) $\mathscr{S}_{S E} \subseteq \mathscr{S}_{S V}$ when $x \mapsto \mathscr{R}_{S V}(x)$ is a single-valued function which has a $C^{1}$-extension to an arbitrary open neighborhood $D_{1} \subset M_{1}$ of $K_{1}$.

Proof. (i) Let $x_{2} \in \mathscr{R}_{S E}\left(x_{1}\right)$ be an arbitrarily fixed element, i.e., $h_{2}\left(x_{1}, y\right) \geq h_{2}\left(x_{1}, x_{2}\right)$ for all $y \in K_{2}$. By definition, we have that

$$
g_{2}\left(\frac{\partial h_{2}}{\partial x_{2}}\left(x_{1}, x_{2}\right), \exp _{x_{2}}^{-1}(y)\right)=\lim _{t \rightarrow 0^{+}} \frac{h_{2}\left(x_{1}, \exp _{x_{2}}\left(t \exp _{x_{2}}^{-1}(y)\right)-h_{2}\left(x_{1}, x_{2}\right)\right.}{t}, \forall y \in K_{2} .
$$

Since $K_{2}$ is geodesic convex, the element $\exp _{x_{2}}\left(t \exp _{x_{2}}^{-1}(y) \in K_{2}\right.$ for every $t \in[0,1]$ whenever $y \in K_{2}$. By the above expression one has that for every $y \in K$,

$$
g_{2}\left(\frac{\partial h_{2}}{\partial x_{2}}\left(x_{1}, x_{2}\right), \exp _{x_{2}}^{-1}(y)\right) \geq 0,
$$

which implies that $\mathscr{R}_{S E}\left(x_{1}\right) \subseteq \mathscr{R}_{S V}\left(x_{1}\right)$ for all $x_{1} \in K_{1}$.

(ii) Since the function $h_{2}\left(x_{1},.\right)$ is convex and of class $C^{1}$, one has

$$
h_{2}\left(x_{1}, y\right)-h_{2}\left(x_{1}, x_{2}\right) \geq g_{2}\left(\frac{\partial h_{2}}{\partial x_{2}}\left(x_{1}, x_{2}\right), \exp _{x_{2}}^{-1}(y)\right)
$$

for all $y \in K_{2}$, see [14]. Taking into account that $x_{2} \in \mathscr{R}_{S V}\left(x_{1}\right)$, one has that

$$
g_{2}\left(\frac{\partial h_{2}}{\partial x_{2}}\left(x_{1}, x_{2}\right), \exp _{x_{2}}^{-1}(y)\right) \geq 0
$$

for all $y \in K_{2}$. Thus, one has $h_{2}\left(x_{1}, y\right)-h_{2}\left(x_{1}, x_{2}\right) \geq 0$ for all $y \in K_{2}$, i.e., $x_{2} \in$ $\mathscr{R}_{S E}\left(x_{1}\right)$.

(iii) The proof is similar to (i).

In the sequel, we shall prove that the elements of the set $\mathscr{R}_{S V}\left(x_{1}\right)$ can be obtained as the fixed points of a carefully choosen map. More precisely, for a fixed $x_{1} \in K_{1}$ and $\alpha>0$, let $\mathscr{F}_{\alpha}^{x_{1}}: K_{2} \rightarrow K_{2}$ be defined by

$$
\mathscr{F}_{\alpha}^{x_{1}}(x)=P_{K_{2}}\left(\exp _{x}\left(-\alpha \frac{\partial h_{2}}{\partial x_{2}}\left(x_{1}, x\right)\right)\right) \text {. }
$$

Theorem 1. Let $\left(M_{1}, g_{1}\right)$ be a Riemannian manifold, and $\left(M_{2}, g_{2}\right)$ be a Hadamard manifold. Let $h_{2}: M_{1} \times M_{2} \rightarrow \mathbf{R}$ be a function of class $C^{1}$ and $K_{i} \subset M_{i}$ closed, geodesic convex sets, $i=1,2$. Let $x_{1} \in K_{1}$. The following statements are equivalent:

(i) $x_{2} \in \mathscr{R}_{S V}\left(x_{1}\right)$;

(ii) $\mathscr{F}_{\alpha}^{x_{1}}\left(x_{2}\right)=x_{2}$ for all $\alpha>0$;

(iii) $\mathscr{F}_{\alpha}^{x_{1}}\left(x_{2}\right)=x_{2}$ for some $\alpha>0$.

Proof. Let us fix $x_{2} \in \mathscr{R}_{S V}\left(x_{1}\right)$ arbitrarily, where $x_{1} \in K_{1}$. By definition, we have that

$$
g_{2}\left(-\alpha \frac{\partial h_{2}}{\partial x_{2}}\left(x_{1}, x_{2}\right), \exp _{x_{2}}^{-1}(y)\right) \leq 0, \forall y \in K_{2}
$$


for all/some $\alpha>0$. Let $\gamma, \sigma:[0,1] \rightarrow M_{2}$ be the unique minimal geodesics defined by

$$
\gamma(t)=\exp _{x_{2}}\left(-t \alpha \frac{\partial h_{2}}{\partial x_{2}}\left(x_{1}, x_{2}\right)\right)
$$

and

$$
\sigma(t)=\exp _{x_{2}}\left(t \exp _{x_{2}}^{-1}(y)\right)
$$

for any fixed $\alpha>0$ and $y \in K_{2}$. Since $K_{2}$ is geodesic convex in $\left(M_{2}, g_{2}\right)$, then $\operatorname{Im} \sigma \subset K_{2}$ and

$$
g_{2}(\dot{\gamma}(0), \dot{\sigma}(0))=g_{2}\left(-\alpha \frac{\partial h_{2}}{\partial x_{2}}\left(x_{1}, x_{2}\right), \exp _{x_{2}}^{-1}(y)\right),
$$

i.e., $\left(M D_{2}\right)$ holds. By the Moskovitz-Dines property, see Proposition 1, one has that

$$
x_{2}=\gamma(0) \in P_{K_{2}}(\gamma(1))=P_{K_{2}}\left(\exp _{x_{2}}\left(-\alpha \frac{\partial h_{2}}{\partial x_{2}}\left(x_{1}, x_{2}\right)\right)\right)=\mathscr{F}_{\alpha}^{x_{1}}\left(x_{2}\right) .
$$

Since $\operatorname{card}\left(\mathscr{F}_{\alpha}^{x_{1}}\left(x_{2}\right)\right)=1$, the proof is complete.

Remark. Note that for all $\alpha>0$,

$$
\mathscr{R}_{S V}\left(x_{1}\right)=\left\{x_{2} \in K_{2}: P_{K_{2}}\left(\exp _{x_{2}}\left(-\alpha \frac{\partial h_{2}}{\partial x_{2}}\left(x_{1}, x_{2}\right)\right)\right)=x_{2}\right\} \text {. }
$$

\section{Follower strategy: existence of equilibria}

\subsection{Compact case}

Theorem 2. (Compact case) Let $\left(M_{i}, g_{i}\right)$ be Hadamard manifolds, $h_{i}: M_{1} \times M_{2} \rightarrow \mathbf{R}$ be functions of class $C^{1}$ and $K_{i} \subset M_{i}$ compact, geodesic convex sets, $i=1,2$. Then the following statements hold:

(i) $\mathscr{R}_{S V}\left(x_{1}\right) \neq \emptyset$ for every $x_{1} \in K_{1}$;

(ii) $\mathscr{S}_{S V} \neq \emptyset$, whenever $\mathscr{R}_{S V}\left(x_{1}\right)$ is a singleton for every $x_{1} \in K_{1}$ and the map $x \mapsto \mathscr{R}_{S V}(x)$ has a $C^{1}$-extension to an arbitrary open neighborhood $D_{1} \subset M_{1}$ of $K_{1}$.

Proof. (i) Fix $x_{1} \in K_{1}$ and $\alpha>0$. Since $K_{2}$ is a Chebishev set and $P_{K_{2}}$ is globally Lipschitz, we see that $\mathscr{F}_{\alpha}^{x_{1}}: K_{2} \rightarrow K_{2}$ is a single-valued continuous function; in particular, $\mathscr{F}_{\alpha}^{x_{1}}: K_{2} \rightarrow K_{2}$ has a closed graph. Moreover, since $K_{2}$ is geodesic convex, it is contractible, thus an acyclic set. Now, we may apply the fixed point theorem of Begle on the compact set $K_{2}$, obtaining that $\mathscr{F}_{\alpha}^{x_{1}}$ has at least a fixed point $x_{2} \in K_{2}$. Due to Theorem $1, x_{2} \in \mathscr{R}_{S V}\left(x_{1}\right)$, which concludes the proof of (i).

(ii) For some $\beta>0$, we introduce the map $\mathscr{G}_{\beta}: K_{1} \rightarrow K_{1}$ defined by

$$
\mathscr{G}_{\beta}(x)=P_{K_{1}}\left(\exp _{x}\left(-\beta \frac{\partial h_{1}}{\partial x}\left(x, \mathscr{R}_{S V}(x)\right)\right)\right) .
$$


Since $\operatorname{card}\left(\mathscr{R}_{S V}(x)\right)=1$ for every $x \in K_{1}$ and the map $x \mapsto \mathscr{R}_{S V}(x)$ has a $C^{1}$ extension to an arbitrary $D_{1} \subset M_{1}$ of $K_{1}$, the function $\mathscr{G}_{\beta}$ is well-defined for every $\beta>0$. By the hypotheses, the function $\mathscr{G}_{\beta}$ is also continuous, thus on account of the Belge fixed point theorem, there exits at least $x_{1} \in K_{1}$ such that $\mathscr{G}_{\beta}\left(x_{1}\right)=x_{1}$. Since $\left(M_{1}, g_{1}\right)$ is a Hadamard manifold where the Moskovitz-Dines property holds, an analogous argument as in Theorem 1 shows that $\mathscr{G}_{\beta}\left(x_{1}\right)=x_{1}$ is equivalent to $x_{1} \in \mathscr{S}_{S V}$. The proof is complete.

\subsection{Non-compact case}

When the strategy sets are non-compact, certain growth assumptions are needed on the payoff functions in order to guarantee the existence of Stackelberg equilibria. We first assume that for some $x_{1} \in K_{1}$ one has

$\left(H_{x_{1}}^{h_{2}}\right)$ There exists $x_{2} \in K_{2}$ such that

$$
\begin{gathered}
L_{x_{1}, x_{2}}=\limsup _{d_{g_{2}}\left(x, x_{2}\right) \rightarrow \infty, x \in K_{2}} \frac{g_{2}\left(\frac{\partial h_{2}}{\partial x_{2}}\left(x_{1}, x\right), \exp _{x}^{-1}\left(x_{2}\right)\right)+g_{2}\left(\frac{\partial h_{2}}{\partial x_{2}}\left(x_{1}, x_{2}\right), \exp _{x_{2}}^{-1}(x)\right)}{d_{g_{2}}\left(x, x_{2}\right)}< \\
<-\left\|\frac{\partial h_{2}}{\partial x_{2}}\left(x_{1}, x_{2}\right)\right\|_{g_{2}} .
\end{gathered}
$$

Theorem 3. Let $\left(M_{1}, g_{1}\right)$ be a Riemannian manifold, and $\left(M_{2}, g_{2}\right)$ be a Hadamard manifold. Let $h_{2}: M_{1} \times M_{2} \rightarrow \mathbf{R}$ be a function of class $C^{1}$ and $K_{i} \subset M_{i}$ closed, geodesic convex sets, $i=1,2$. Let $x_{1} \in K_{1}$ and assume that hypothesis $\left(H_{x_{1}}^{h_{2}}\right)$ holds true. Then $\mathscr{R}_{S V}\left(x_{1}\right) \neq \emptyset$.

Proof. Let $E_{0} \in \mathbf{R}$ such that

$$
L_{x_{1}, x_{2}}<-E_{0}<-\left\|\frac{\partial h_{2}}{\partial x_{2}}\left(x_{1}, x_{2}\right)\right\|_{g_{2}} .
$$

On account of hypothesis $\left(H_{x_{1}}^{h_{2}}\right)$ there exists $R>0$ large enough such that for every $x \in K_{2}$ with $d_{g_{2}}\left(x, x_{2}\right) \geq R$, we have

$$
g_{2}\left(\frac{\partial h_{2}}{\partial x_{2}}\left(x_{1}, x\right), \exp _{x}^{-1}\left(x_{2}\right)\right)+g_{2}\left(\frac{\partial h_{2}}{\partial x_{2}}\left(x_{1}, x_{2}\right), \exp _{x_{2}}^{-1}(x)\right) \leq-E_{0} d_{g_{2}}\left(x, x_{2}\right) .
$$

Clearly, one may assume that $K_{2} \cap \bar{B}_{g_{2}}\left(x_{2}, R\right) \neq \emptyset$. In particular, from (3) and (2), for every $x \in K_{2}$ with $d_{g_{2}}\left(x, x_{2}\right) \geq R$, the above relation yields

$$
\begin{aligned}
g_{2}\left(\frac{\partial h_{2}}{\partial x_{2}}\left(x_{1}, x\right), \exp _{x}^{-1}\left(x_{2}\right)\right) \leq & -E_{0} d_{g_{2}}\left(x, x_{2}\right) \\
& +\left\|\frac{\partial h_{2}}{\partial x_{2}}\left(x_{1}, x_{2}\right)\right\|_{g_{2}}\left\|\exp _{x_{2}}^{-1}(x)\right\|_{g_{2}} \\
= & \left(-E_{0}+\left\|\frac{\partial h_{2}}{\partial x_{2}}\left(x_{1}, x_{2}\right)\right\|_{g_{2}}\right) d_{g_{2}}\left(x, x_{2}\right) \\
< & 0 .
\end{aligned}
$$


Let $K_{R}=K_{2} \cap \bar{B}_{g_{2}}\left(x_{2}, R\right)$. It is clear that $K_{R}$ is a geodesic convex, compact subset of $\left(M_{2}, g_{2}\right)$. Due to Theorem 2, we immediately have that there exists $\tilde{x}_{2} \in K_{R}$ such that

$g_{2}\left(\frac{\partial h_{2}}{\partial x_{2}}\left(x_{1}, \tilde{x}_{2}\right), \exp _{\tilde{x}_{2}}^{-1}(y)\right) \geq 0$ for all $y \in K_{R}$.

Note that $d_{g_{2}}\left(\tilde{x}_{2}, x_{2}\right)<R$. By assuming the contrary, from (6) with $x=\tilde{x}_{2}$ we have that

$$
g_{2}\left(\frac{\partial h_{2}}{\partial x_{2}}\left(x_{1}, \tilde{x}_{2}\right), \exp _{\tilde{x}_{2}}^{-1}\left(x_{2}\right)\right)<0,
$$

by contradicting relation (7).

Let us choose $z \in K_{2}$ arbitrarily. From the fact that $d_{g_{2}}\left(\tilde{x}_{2}, x_{2}\right)<R$, for $\varepsilon>0$ small enough, the element $y=\exp _{\tilde{x}_{2}}\left(\varepsilon \exp _{\tilde{x}_{2}}^{-1}(z)\right)$ belongs both to $K_{2} \cap \bar{B}_{g_{2}}\left(x_{2}, R\right)=K_{R}$. By replacing $y$ into (7), we obtain that

$$
g_{2}\left(\frac{\partial h_{2}}{\partial x_{2}}\left(x_{1}, \tilde{x}_{2}\right), \exp _{\tilde{x}_{2}}^{-1}(z)\right) \geq 0 .
$$

Since $z \in K_{2}$ is arbitrarily fixed, one has that $\tilde{x}_{2} \in \mathscr{R}_{S V}\left(x_{1}\right)$, which ends the proof. $\triangle$

In the sequel, we are dealing with another class of functions. For a fixed $x_{1} \in K_{1}$, $\alpha>0$ and $0<\rho<1$ we introduce the hypothesis:

$$
\begin{aligned}
\left(H_{x_{1}}^{\alpha, \rho}\right): \quad d_{g_{2}} & \left(\exp _{x}\left(-\alpha \frac{\partial h_{2}}{\partial x_{2}}\left(x_{1}, x\right)\right), \exp _{y}\left(-\alpha \frac{\partial h_{2}}{\partial x_{2}}\left(x_{1}, y\right)\right)\right) \leq \\
\leq & (1-\rho) d_{g_{2}}(x, y) \text { for all } x, y \in K_{2} .
\end{aligned}
$$

For fixed $x_{1} \in K_{1}$ and $\alpha>0$, we consider the following two dynamical systems:

(a) let $(D D S)_{x_{1}}$ be the discrete differential system in the form

$$
\left\{\begin{array}{l}
y_{n+1}=\mathscr{F}_{\alpha}^{x_{1}}\left(P_{K_{2}}\left(y_{n}\right)\right), \quad n \geq 0 \\
y_{0} \in M_{2}
\end{array}\right.
$$

(b) Let $(C D S)_{x_{1}}$ be the continuous differential system in the form

$$
\left\{\begin{array}{l}
\frac{d y}{d t}=\exp _{y(t)}^{-1}\left(\mathscr{F}_{\alpha}^{x_{1}}\left(P_{K_{2}}(y(t))\right)\right), \\
y(0)=x_{2} \in M_{2}
\end{array}\right.
$$

The main result of the present section is the following theorem.

Theorem 4 (Non-compact case). Let $\left(M_{1}, g_{1}\right)$ be a Riemannian manifold, and $\left(M_{2}, g_{2}\right)$ be a Hadamard manifold. Let $h_{2}: M_{1} \times M_{2} \rightarrow \mathbf{R}$ be a function of class $C^{1}$ and $K_{i} \subset M_{i}$ closed, geodesic convex sets, $i=1,2$. Let $x_{1} \in K_{1}$ and assume that hypothesis $\left(H_{x_{1}}^{\alpha, \rho}\right)$ holds true. Then $\mathscr{R}_{S V}\left(x_{1}\right)$ is a singleton and both dynamical systems, $(D D S)_{x_{1}}$ and $(C D S)_{x_{1}}$, exponentially converge to the unique element of $\mathscr{R}_{S V}\left(x_{1}\right)$. 
Proof. Since $\left(M_{2}, g_{2}\right)$ is a Hadamard manifold, for the geodesic convex set $K_{2} \subset M_{2}$ we have that $P_{K_{2}}$ is non-expansive. Therefore, by $\left(H_{x_{1}}^{\alpha, \rho}\right)$, one has for every $x, y \in K_{2}$ that

$$
\begin{aligned}
d_{g_{2}} & \left(\mathscr{F}_{\alpha}^{x_{1}}(x), \mathscr{F}_{\alpha}^{x_{1}}(y)\right) \\
\quad & =d_{g_{2}}\left(P_{K_{2}}\left(\exp _{x}\left(-\alpha \frac{\partial h_{2}}{\partial x_{2}}\left(x_{1}, x\right)\right)\right), P_{K_{2}}\left(\exp _{y}\left(-\alpha \frac{\partial h_{2}}{\partial x_{2}}\left(x_{1}, y\right)\right)\right)\right) \\
& \leq d_{g_{2}}\left(\exp _{x}\left(-\alpha \frac{\partial h_{2}}{\partial x_{2}}\left(x_{1}, x\right)\right), \exp _{y}\left(-\alpha \frac{\partial h_{2}}{\partial x_{2}}\left(x_{1}, y\right)\right)\right) \\
& \leq(1-\rho) d_{g_{2}}(x, y) .
\end{aligned}
$$

Consequently, the function $\mathscr{F}_{\alpha}^{x_{1}}$ is a $(1-\rho)$-contraction on $K_{2}$.

(a) The system $(D D S)_{x_{1}}$. We shall apply the Banach fixed point theorem to the function $\mathscr{F}_{\alpha}^{x_{1}}: K_{2} \rightarrow K_{2}$, by guaranteeing the existence of the unique fixed point of $\mathscr{F}_{\alpha}^{x_{1}}$ for every $x_{1} \in K_{1}$. Moreover, every iterated sequence in the dynamical system $(D D S)_{x_{1}}$ converges exponentially to the unique fixed point $x_{2} \in K_{2}$ of $\mathscr{F}_{\alpha}^{x_{1}}$. Due to Theorem 1 the set $\mathscr{R}_{S V}\left(x_{1}\right)$ is a singleton with the element $x_{2}$. Moreover, for all $k \in \mathbf{N}$ we have that

$$
d_{g_{2}}\left(y_{k}, x_{2}\right) \leq \frac{(1-\rho)^{k}}{\rho} d_{g_{2}}\left(y_{1}, y_{0}\right)
$$

(b) The system $(C D S)_{x_{1}}$. First of all, standard ODE theory shows that $(C D S)_{x_{1}}$ has a (local) solution in $[0, T)$. We actually prove that $T=+\infty$. To see this fact, we assume that $T<+\infty$, and we introduce the Lyapunov function which has the form

$$
h_{x_{1}}(t)=\frac{1}{2} d_{g_{2}}\left(y(t), x_{2}\right)^{2} .
$$

Note that for a.e. $t \in[0, T)$, we have

$$
\begin{aligned}
\frac{d}{d t} h_{x_{1}}(t)= & -g_{2}\left(\exp _{y(t)}^{-1}\left(x_{2}\right), \frac{d y}{d t}\right) \\
= & -g_{2}\left(\exp _{y(t)}^{-1}\left(x_{2}\right), \exp _{y(t)}^{-1}\left(\mathscr{F}_{\alpha}^{x_{1}}\left(P_{K_{2}}(y(t))\right)\right)\right) \\
= & -g_{2}\left(\exp _{y(t)}^{-1}\left(x_{2}\right), \exp _{y(t)}^{-1}\left(\mathscr{F}_{\alpha}^{x_{1}}\left(P_{K_{2}}(y(t))\right)\right)-\exp _{y(t)}^{-1}\left(x_{2}\right)\right) \\
& \left.-g_{2}\left(\exp _{y(t)}^{-1}\left(x_{2}\right), \exp _{y(t)}^{-1}\left(x_{2}\right)\right)\right) \\
\leq & \left\|\exp _{y(t)}^{-1}\left(\mathscr{F}_{\alpha}^{x_{1}}\left(P_{K_{2}}(y(t))\right)\right)-\exp _{y(t)}^{-1}\left(x_{2}\right)\right\|_{g_{2}}\left\|\exp _{y(t)}^{-1}\left(x_{2}\right)\right\|_{g_{2}} \\
& -\left\|\exp _{y(t)}^{-1}\left(x_{2}\right)\right\|_{g_{2}}^{2} .
\end{aligned}
$$

By using the fact that $\left(M_{2}, g_{2}\right)$ is a Hadamard manifold, a Rauch comparison theorem and further straightforward estimates show that

$$
\left\|\exp _{y(t)}^{-1}\left(\mathscr{F}_{\alpha}^{x_{1}}\left(P_{K_{2}}(y(t))\right)\right)-\exp _{y(t)}^{-1}\left(x_{2}\right)\right\|_{g_{2}} \leq d_{g_{2}}\left(\mathscr{F}_{\alpha}^{x_{1}}\left(P_{K_{2}}(y(t))\right), x_{2}\right) .
$$


Therefore, by (3) and the non-expansiveness of $P_{K_{2}}$, we have

$$
\begin{aligned}
\frac{d}{d t} h_{x_{1}}(t) & \leq d_{g_{2}}\left(\mathscr{F}_{\alpha}^{x_{1}}\left(P_{K_{2}}(y(t))\right), x_{2}\right) d_{g_{2}}\left(y(t), x_{2}\right)-d_{g_{2}}\left(y(t), x_{2}\right)^{2} \\
& =d_{g_{2}}\left(\mathscr{F}_{\alpha}^{x_{1}}\left(P_{K_{2}}(y(t))\right), \mathscr{F}_{\alpha}^{x_{1}}\left(x_{2}\right)\right) d_{g_{2}}\left(y(t), x_{2}\right)-d_{g_{2}}\left(y(t), x_{2}\right)^{2} \\
& \leq(1-\rho) d_{g_{2}}\left(P_{K_{2}}(y(t)), x_{2}\right) d_{g_{2}}\left(y(t), x_{2}\right)-d_{g_{2}}\left(y(t), x_{2}\right)^{2} \\
& \leq(1-\rho) d_{g_{2}}\left(y(t), x_{2}\right)^{2}-d_{g_{2}}\left(y(t), x_{2}\right)^{2} \\
& =-\rho d_{g_{2}}\left(y(t), x_{2}\right)^{2} \\
& =-2 \rho h_{x_{1}}(t), \text { a.e. } t \in[0, T) .
\end{aligned}
$$

Therefore, one has

$$
\frac{d}{d t}\left[h_{x_{1}}(t) e^{2 \rho t}\right]=\left(\frac{d}{d t} h_{x_{1}}(t)+2 \rho h_{x_{1}}(t)\right) e^{2 \rho t} \leq 0 .
$$

In particular, the function $t \mapsto h_{x_{1}}(t) e^{2 \rho t}$ is non-increasing; therefore, for all $t \in$ $[0, T)$ one has that $h_{x_{1}}(t) e^{2 \rho t} \leq h_{x_{1}}(0)$. Consequently, $t \mapsto y(t)$ can be extended beyond $T$, contradicting our assumption. Therefore, $T=+\infty$.

The above estimate gives that for every $t \geq 0, h_{x_{1}}(t) \leq h_{x_{1}}(0) e^{-2 \rho t}$. In particular, it yields that

$$
d_{g_{2}}\left(y(t), x_{2}\right) \leq d_{g_{2}}\left(y_{0}, x_{2}\right) e^{-\rho t} .
$$

The proof is concluded.

Remark. Assume that $M_{i}=\mathbf{R}^{m_{i}}, i=1,2$ and $\frac{\partial f_{2}}{\partial x_{2}}\left(x_{1}, \cdot\right)$ is an $\lambda$-Lipschitz and $\sigma$-strictly monotone function for some $x_{1} \in K_{1}$, i.e.,

- $\left\|\frac{\partial f_{2}}{\partial x_{2}}\left(x_{1}, x\right)-\frac{\partial f_{2}}{\partial x_{2}}\left(x_{1}, y\right)\right\| \leq \lambda\|x-y\|$,

- $\left\langle\frac{\partial f_{2}}{\partial x_{2}}\left(x_{1}, x\right)-\frac{\partial f_{2}}{\partial x_{2}}\left(x_{1}, y\right), x-y\right\rangle \geq \sigma\|x-y\|^{2}, \forall x, y \in \mathbf{R}^{m_{2}}$.

In this case, $\left(H_{x_{1}}^{\varepsilon, \rho}\right)$ holds true with

$$
0<\varepsilon<\frac{\sigma-\sqrt{\left(\sigma^{2}-\lambda^{2}\right)_{+}}}{\lambda^{2}}
$$

and

$$
\rho=1-\sqrt{1-2 \varepsilon \sigma+\varepsilon^{2} \lambda^{2}} \in(0,1) .
$$

Remark. Very recently, Kristály and Repovs [6] proved that the Moskovitz-Dines property on a generic Riemannian manifold implies the non-positiveness of the sectional curvature. Consequently, in order to develop the aforementioned results on 'curved' spaces, the non-positiveness of the sectional curvature seems to be a natural requirement.

Remark. By following the non-smooth critical point theory of Szulkin [12], it would be interesting to guarantee not only the existence of Stackelberg equilibrium points but also some multiplicity results. Here, the indicator function of geodesic convex 
sets as well as the Fréchet subdifferential of the indicator function (as the normal cone to the geodesic convex set) seem to play crucial roles which will be investigated in a forthcoming paper.

\section{Acknowledgement}

The work of Szilárd Nagy is supported by the grant PCCE-55/2008.

\section{References}

[1] E. Cavazzuti, M. Pappalardo, M. Passacantando, Nash equilibria, variational inequalities, and dynamical systems, J. Optim. Theory Appl. 114 (2002), no. 3, 491-506.

[2] M. P. do Carmo, Riemannian Geometry, Birkhäuser, Boston, 1992.

[3] S. Grognet, Théorème de Motzkin en courbure négative, Geom. Dedicata 79 (2000), 219-227.

[4] A. Kristály, Location of Nash equilibria: a Riemannian geometrical approach, Proc. Amer. Math. Soc. 138 (2010), 1803-1810.

[5] A. Kristály, V. Rădulescu, Cs. Varga, Variational Principles in Mathematical Physics, Geometry, and Economics, Cambridge University Press, Encyclopedia of Mathematics and its Applications, No. 136, 2010.

[6] A. Kristály, D. Repovs, Metric Projections versus Non-Positive Curvature, preprint, 2013.

[7] C. Li, G. López, V. Martín-Márquez, Monotone vector fields and the proximal point algorithm on Hadamard manifolds, J. Lond. Math. Soc. (2) 79 (2009), no. 3, 663-683.

[8] D. Moskovitz, L.L. Dines, Convexity in a linear space with an inner product, Duke Math. J. 5 (1939) 520-534.

[9] Sz. Nagy, Stackelberg equilibria via variational inequalities and projections, J. Global Optimization, in press (2013).

[10] S.Z. Németh, Variational inequalities on Hadamard manifolds, Nonlinear Analysis 52 (2003), 1491-1498.

[11] J.-S. Pang, M. Fukushima, Quasi-variational inequalities, generalized Nash equilibria, and multi-leader-follower games, CMS 2(2005), 21-56. DOI: 10.1007/s10287-004-0010-0

[12] A. Szulkin, Minimax prin- ciples for lower semicontinuous functions and applications to nonlinear boundary value problems. Ann. Inst. H. Poincaré Anal. Non Linéaire 3 (1986), no. 2, 77-109.

[13] R. Walter, On the metric projection onto convex sets in Riemannian spaces, Arch. Math. (Basel) 25 (1974), 91-98. 
[14] C. Udrişte, Convex Functions and Optimization Methods on Riemannian Manifolds, Mathematics and its Applications, 297. Kluwer Academic Publishers Group, Dordrecht, 1994.

[15] Y.S. Xia, J. Wang, On the stability of globally projected dynamical systems, J. Optim. Theory Appl. 106 (2000), no. 1, 129-150.

[16] J. Zhang, B. Qu, N. Xiu, Some projection-like methods for the generalized Nash equilibria, Comput. Optim. Appl. 45 (2010), 89-109. 